\title{
Comparative Analysis of Utilization Based Hybrid Overlay for Live Video Streaming in P2P Network
}

\author{
Kunwar Pal ${ }^{1}$, M.C. Govil ${ }^{2}$, Mushtaq Ahmed ${ }^{3}$ \\ ${ }^{1,2,3}$ Department of Computer Science and Engineering, Malaviya National Institute of Technology Jaipur, India
}

\begin{abstract}
With a sudden surge in demand for live video streaming using peer to peer $(P 2 P)$ network, there has been a rise of traffic in media transmission applications that employ this $P 2 P$ approach. $P 2 P$ is a decentralized media communication method which is more cost effective, and scalable. However, the traditional peer to peer network architecture has several limitations, and overlay construction is one of them. The two different unstructured overlay approaches like tree and mesh have already been discussed. This paper gives a detailed comparison of various overlay approaches in P2P networks. We have also proposed and implemented a utilization based hybrid overlay approach for the P2P network. The two crucial parameters which are primarily considered while overlay construction is: peer upload bandwidth and peer resource utilization. For the purpose of verifying our approach, we have done a comparative analysis of the proposed utilization based approach with the popular DenaCast approach. The different QoS parameters like start-up delay, playback delay, end to end delay, frame loss ratio are used for verifying our approach. The simulation results presented in this paper support the proposed approach.
\end{abstract}

Keywords: P2P, Peer to Peer network, Live video streaming, Resource utilization, Content Delivery Network, ISP traffic.

\section{Introduction}

In the last couple of years, an exponential increase has been witnessed in the number of users that has contributed to a steep rise in the requirement of online video streaming. Some popular user applications of online video streaming are YouTube [1], NetTv [2], IPTV [3] etc. According to a study conducted by Cisco[4], it has been estimated that till 2019, 80-90\% of data transmission over the internet will be only due to video transmission. Complexity and cost of the server side increases due to the exponential increase of users for video streaming in traditional client-server architecture. Peer to Peer (P2P) computing can be a solution to the issues in traditional client-server architecture. It is a distributed computation mechanism. Due to its properties like scalability, easy to maintain, low cost and complexity at the server side, the popularity of P2P network is increasing rapidly. Resources of receiver peers are also used for the improvement of the network, and thus overall resource utilization of network is improved. P2P network is more reliable and fault tolerance system as compared to traditional client-server architecture. A combination of both traditional client-server and the P2P network is more efficient and provides the solution to the problems of both the architectures [5]. This integration of the P2P network approach with live video streaming has been implemented in CoolStreaming [6]. It is a milestone work in the field of live video streaming for P2P networks. This approach using CoolStreaming for content distribution is the same as in BitTorrent[7]. The basic idea with design issues and implementation of CoolStreaming are discussed in detail by SusuXie et al. [8]. An improvement of CoolStreaming, its design theory, and practical implementation is discussed by [9], where churn is described as a crucial factor in P2P live streaming. Overlay construction, scheduling scheme, selfish peer and the flash crowd are some of the factors which affect the P2P network. Overlay construction is a crucial part of the P2P network, and the network performance is directly affected by the overlay design of the network. In this paper, we will focus on the different overlay construction approaches and provide a new utilization based overlay in $\mathrm{P} 2 \mathrm{P}$ network using the different parameters like upload bandwidth and the ratio of utilization of peer. 


\section{Related Work}

Traditionally, overlay construction in the P2P network can be classified into; tree overlay and mesh overlay.

\subsection{Tree Overlay}

Tree overlay is a simple and efficient approach that supports multicasting. It is less complex and easy to maintain compared to the other overlay approaches available in the P2P network. Source or video server is available at the zero level, and a hierarchy of peers is created that comprises of multiple levels. As the number of peers in the network increases; the number of levels in the tree overlay also increases. A peer can only connect to one parent at a time, and a parent peer can have any number of children in a simple tree overlay. So, parent peers forward the video content to all its available child peers in the tree overlay. The child peer can also do the same for their children, so the media content is reachable to all the peers available in the network. Some of the examples of tree-based overlay are ESM and NICE [10]. Parent-child relationship between the peers in tree overlay decreases the start-up delay which is not the case in other overlay structures in the P2P network. The leaf peers can only download the media content from their immediate parents, but they cannot upload the content in the network so their upload bandwidth is not used and the overall performance of the network drops.

\subsection{Mesh Overlay}

A solution to the tree overlay problem can be given by using the mesh-based overlay. Peer in mesh-based overlay follow the property of complete mesh. In the complete mesh approach, each peer is connected to every other peer available in the network. Push based scheduling scheme is not used in the mesh-based overlay. Push based scheduling with mesh-based overlay provides the replication of media content in the network. Thus, peers can receive the media content from more than one peer. So, if the peer is dynamic in nature and leaves the network frequently, in that case, the child peer can still retrieve the media content from the other peers. The impact of a dynamic peer is less in mesh overlay as compared to that in tree overlay. This makes the mesh overlay network more reliable in comparison to the tree overlay network. Peers who are lower in the hierarchy in the mesh-based overlay can also upload the content. So the bandwidth utilization of the peer lower in the hierarchy is higher as compared to that in tree overlay. This affects the overall resource utilization of P2P network [11]. Due to the frequent exchange of notifications mesh overlay suffers from efficiency latency tradeoff. Chainsaw, CoolStreaming, Bullet, and Anysee, are the examples of mesh-based overlay [12]. Bandwidthbottleneck and content-bottleneck are the two main problems which arise while using live video streaming in a P2P network. P2P live video streaming using mesh-based overlay is discussed in PRIME in detail [13]. A solution to the bandwidth and content-bottleneck problems is given in this PRIME approach. For contentbottleneck, efficient pattern delivery approach is used, and for bandwidth-bottleneck, the bandwidth degree approach [14] is used.

\subsection{Hybrid Overlay}

Traditionally, the unstructured overlay can be classified into tree and mesh overlay. Maintenance of tree overlay is easy, control overhead of tree overlay is minimized, start-up delay and transmission delay are also less in tree overlay as compare to mesh overlay. But maintenance of tree overlay increases with an increase in the number of dynamic peers in the P2P network as they are placed higher in the hierarchy. While mesh overlay is more reliable and scalable, resource utilization of mesh overlay is more compared to that for tree overlay. And thus, mesh overlay is more complicated. Both the overlay approaches do not provide an optimal solution to the overlay construction problem in the P2P network. So, some authors have tried to combine both these overlay approaches and provide a new aspect to the overlay construction problem. Q. Huang et al. [15] defined a novel approach for hybrid overlay construction. This approach is a combination of both the tree and mesh overlay approaches, tree overlay is used for control packet transmission while mesh overlay is used for transfer of media packets. For tree overlay, geographical location of the peer is used and for mesh overlay layered peer selection mechanism is used. The efficiency of each peer is calculated periodically, and inefficient peers are removed 
from the overlay. Only geographical location of a peer is used in the approach, and different crucial parameters like stability and bandwidth of the peer are not considered.

An another approach which uses similar bandwidth range to create a tree overlay is discussed in HyPO [12]. In a geographical place, the peers that are in the same bandwidth range create the tree overlay. The depth of the tree plays a significant role in tree overlay. An even distribution of peers is done so that the depth of the tree is minimized. Further, a mesh is created between the peers that are at the same geographical location. Transmission time taken by the approach is less as compared to the other approaches due to the depth of the tree. The control overhead of the approach is also minimized. There is no clear criterion to distinguish between using the tree or mesh overlay in this approach. Also, the upload bandwidth of stable peers is not fully utilized in the approach. Bandwidth utilization of the peers in the P2P network is a prime consideration. To solve this issue, a Hybrid Live P2P Streaming Protocol (HLPSP) is described by the author to solve the problem of hybrid overlay network [11]. Overlay creation uses the upload bandwidth of each peer. So the peer that have maximum upload bandwidth should be higher in the hierarchy. Source peer is at the level $O$ with the highest bandwidth. Simulation analysis of the approach is done with the DenaCast approach (an enhanced version of CoolStreaming). Through the simulation, authors show that the HLPSP provides better results compared to the DenaCast approach. HLPSP consider the upload bandwidth of peers for overlay creation, other crucial factors like the stability of each peer, geographical location are not considered. A new hybrid overlay creation is discussed in our previous work [16], which consider all different crucial factors which are discussed above for overlay construction. This approach is a hybrid overlay combination of both tree and mesh overlay. Tree overlay is created using the stability of peers and mesh is created only at same level according to the upload bandwidth, age, and geographical location. Simulation result of the approaches shows that start up delay, playback delay, and the end to end delay is less for the approach as compared to that for DenaCast approach [17].

\section{Utilization Based Overlay}

The above discussion shows that the upload bandwidth of the peers is a prime factor in the P2P overlay network. The upload bandwidth of peer plays a significant role but if a peer doesn't have good connectivity to other peers or peer doesn't upload sufficient amount of data to other peers then its upload bandwidth doesn't remain as efficient. So, not just the upload bandwidth of the peer but also the bandwidth utilization of each peer in the overlay also plays a significant role. In this paper, we will provide a new utilization-based approach for creating an overlay in the $\mathrm{P} 2 \mathrm{P}$ network. The peers that utilize their resources more are given priority instead of those peers that have more upload bandwidth but properly don't utilize their resources. This overlay is a hybrid overlay, and it leverages the properties of both tree and mesh overlays. Procedure to be followed when a new peer enters the network is described in Algorithm 1.

\section{Algorithm 1[A1]: Procedure for New peer i}

1. $R E Q_{i}^{T}<\mathrm{B}_{\mathrm{TU}}^{\mathrm{i}}, \mathrm{C}_{\mathrm{id}}, \mathrm{G}_{\mathrm{i}}>$

2. If Request is new goto 3 else 4 .

3. Position find for new peer $P_{i}\left(\mathrm{~B}_{\mathrm{TU}}^{\mathrm{i}}, \mathrm{G}_{\mathrm{i}}\right)$ [A2]

4. Neighbour/Parent List Creation $L_{i}$. [A3]

5. $\operatorname{RES}_{T}^{i}\left(L_{i}\right)$

6. Check update Periodically

7. $C \_r e q_{i}^{j}$ where $\left(\mathrm{j} \subseteq L_{i}\right)$

8. $C_{-} r e s_{j}^{i}$ (Positive/Negative)

9. if $\left(C_{-} r e s_{j}^{i}\right)$ goto 10 else 7 for new $\mathrm{j}$ from $L_{i}$

10. According to RTT time finds the best response

11. If continue step (12) else leave the network.

12. Periodically update status to Tracer goto (2). 
When a new peer enters in the P2P network, it will follow the procedure as shown in algorithm 1. The new peer $\mathrm{i}$ sends the request to tracker $\mathrm{T}$. This request is a tuple which consists of three variables < $\mathrm{B}_{\mathrm{TU}}^{\mathrm{i}}, \mathrm{C}_{\mathrm{id}}, \mathrm{G}_{\mathrm{i}}>$ where $\mathrm{B}_{\mathrm{TU}}^{\mathrm{i}}$ is the Total upload bandwidth of peer $\mathrm{i}, \mathrm{C}_{\mathrm{id}}$ is the content id of media which peer $\mathrm{i}$ wants to view, $G_{i}$ is the geographical location of peer $i$. Tracker receives the requested tuple and calculates the level for peer i in the overlay, which is shown in Algorithm 2.

\section{Algorithm 2[A2]: Level Find $\left(B_{T U}^{i}, G_{i}\right)$}

1. Find $\mathrm{B}_{\mathrm{TU}}^{\mathrm{S}}$

2. Find Range $\mathrm{R}$ of network

3. Search location according to $\left(\mathrm{C}_{\mathrm{id}}, \mathrm{G}_{\mathrm{i}}\right)$

4. $\mathrm{L}_{-} \mathrm{vel}_{\mathrm{i}}=\operatorname{Ceil}\left[\left\{\mathrm{B}_{\mathrm{TU}}^{\mathrm{S}}-\mathrm{B}_{\mathrm{TU}}^{\mathrm{i}}\right\} / \mathrm{R}\right]$;

5. Return L_vel ${ }_{i}$;

Algorithm 2 finds the level of peers in P2P overlay by using the geographical location and upload bandwidth. After finding the level for peer i, tracker finds the list of best available peers for peer i. From this list, peer i can choose an appropriate neighbours/parent. Algorithm 3 is used for list creation by the tracker.

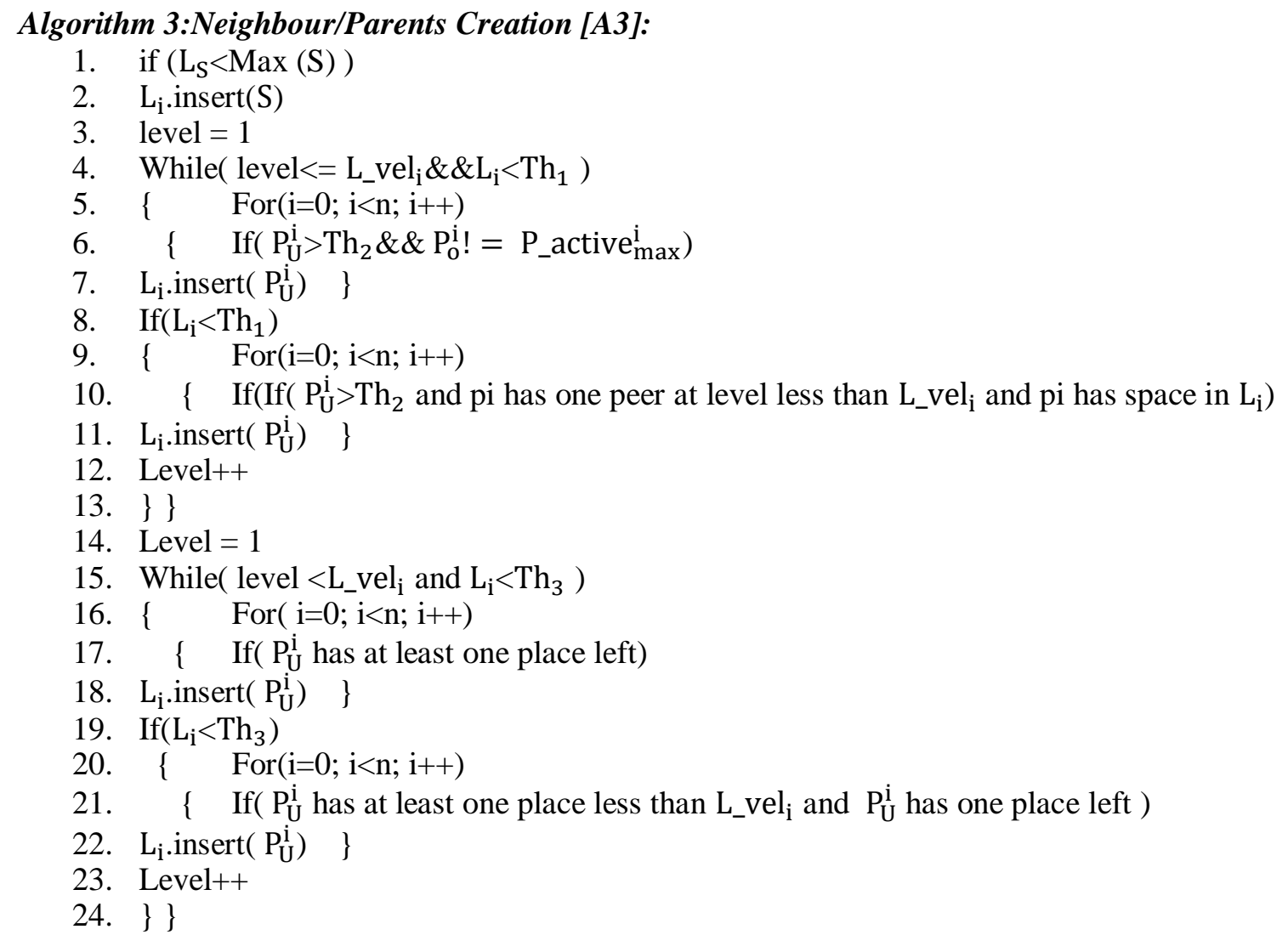

For creating the list of possible parents, tracker uses the level of each peer and utilization of each peer. The server is considered at the highest level in the overlay. So priority is given to the server (line 1-2, algorithm 3) but if the peer is lower in the overlay, then update also takes place (line 11-20, algorithm 3). The list contains the peers only for lower levels (server is at the 0 level and as the height of the tree increases the level also increases). And for each level, only those peers are added to the list that has utilization value greater than the threshold $\left(\mathrm{Th}_{2}\right)$. Tracker adds the new peer to the list, if there is sufficient space in the list $\left(\mathrm{Th}_{1}\right)$ and if the level of the old peer is less than the level of new peer (line 4-13, algorithm 4). For calculating the utilization of each peer, only the data of its last three transactions is considered. And for a new peer that comes for the first time in the network, the average utilization value of the network is assigned to that peer. The overall overlay is created 
in such a way that the peers which have sufficient upload bandwidth are placed above in the hierarchy. For parent selection only those peers are considered which have a higher utilization ratio so; a new peer can receive the media content as quickly as possible.

After creating the list of possible parents/neighbour, tracker sends that list to requesting peer i. Peer i sends the connection request to neighbour peer from the list. Peer, i can send the connection request to more than one peer, and according to RTT (Round Trip Time) of the responding peer, it chooses the parent peer (line 5-10, Algorithm 1). Tracker provides the list according to the utilization, upload bandwidth, and geographical location; however, RTT time gives the idea of real-time congestion in the network. The nomenclature used in the paper is defined in Table 1.

TABLE1: Parameter Used in Algorithm.

\begin{tabular}{cll}
\hline \hline S. No & \multicolumn{1}{c}{ Parameter } & \multicolumn{1}{c}{ Meaning } \\
\hline \hline 1. & Max $(\mathrm{S})$ & Maximum number of peer that can connect to server \\
2. & $\mathrm{P}_{-} a c t i v \mathrm{e}_{\max }^{\mathrm{i}}$ & Maximum number of active peers \\
3. & $\mathrm{Th}_{1}$ & Maximum passive peers of new peer $=\mathrm{B}_{\mathrm{u}} /$ frame size \\
4. & $\mathrm{Th}_{2}$ & Average Utilization of Network \\
5. & $\mathrm{~L}_{-} \mathrm{vel}_{\mathrm{i}}$ & Level of peer i \\
6. & $\mathrm{Th}_{3}$ & $90 \%$ of maximum possible neighbours \\
7. & $\mathrm{P}_{\mathrm{U}}^{\mathrm{i}}$ & Utilization ratio of peer $\mathrm{i},\{$ Uploaded bandwidth / total bandwidth $\}$ \\
8. & $\mathrm{~L}_{\mathrm{i}}$ & list for peer i \\
9. & $\mathrm{~B}_{\mathrm{u}[\mathrm{pk}]}$ & Total upload bandwidth of peer $\mathrm{k}$ \\
10. & $R E S_{T}^{i}\left(L_{i}\right)$ & Response from Tracker to peer $\mathrm{i}$ \\
11. & $\mathrm{C}_{-} r \mathrm{rq}_{\mathrm{i}}^{\mathrm{j}}$ & Connection request from peer $\mathrm{i}$ to $\mathrm{j}$ \\
\hline \hline
\end{tabular}

In this paper, we simulate the approach and provide the results. Different parameters like start-up delay, the end to end delay, playback delay, frame loss ratio and packet drop due to destination unreachable are used to verify the result.

\section{Simulation and Result}

\subsection{Simulation Setup:}

This Utilization based overlay approach is implemented using the OverSim simulator. OverSim is a P2P network simulator and open source overlay for OMNET++ simulation environment (OS). For exchanging and processing network messages Discrete event simulation (DES) is used in OverSim [18]. Two different modules are employed in OverSim and processing between the modules is done using the $\mathrm{C}++$ language and for topology creation, the Network Descriptor (NED) language is used. The parameters which are used in our simulation are given in Table 2.

TABLE2: Simulation Parameters and Values.

\begin{tabular}{clc}
\hline \hline S. No & \multicolumn{1}{c}{ Parameter } & Value \\
\hline \hline 1. & Simulation Duration & $500 \mathrm{~s}$ \\
2. & Buffer Map Exchange period & $1 \mathrm{~s}$ \\
3. & Average Video Bit Rate & $512 \mathrm{Kbps}$ \\
4. & Source Number & 3 \\
5. & Video Codec & MPEG4 Part I \\
6. & Chunk Size & 5 Frames \\
7. & Average Chunk Length & $130 \mathrm{~Kb}$ \\
8. & Number of Runs & 10 \\
9. & Neighbour Notification Period & $2 \mathrm{~s}$ \\
10. & Maximum Number of levels & 6 \\
\hline \hline
\end{tabular}

\subsection{Simulation Results:}

Utilization-based overlay affects the different QoS parameters in P2P live video streaming. Start-up delay plays a significant role in QoS of P2P live video streaming. Start-up delay is directly affected by the overlay. In 
the utilization based overlay, peers form an overlay according to their bandwidth utilization. So, peers that are using their upload bandwidth more are getting higher priority, and there is a high probability of them being selected as parents to other peers. This directly affects the start-up delay. If a peer chooses those peers (utilized peers) as their parents then start-up delay is less compared to the DenaCast approach (an enhanced version of CoolStreaming). Initially, start-up delay for both the approaches is approximately same, but as the number of peers increases, start-up delay for DenaCast approach becomes more as compared to that for utilization based overlay as shown in Fig. 1.

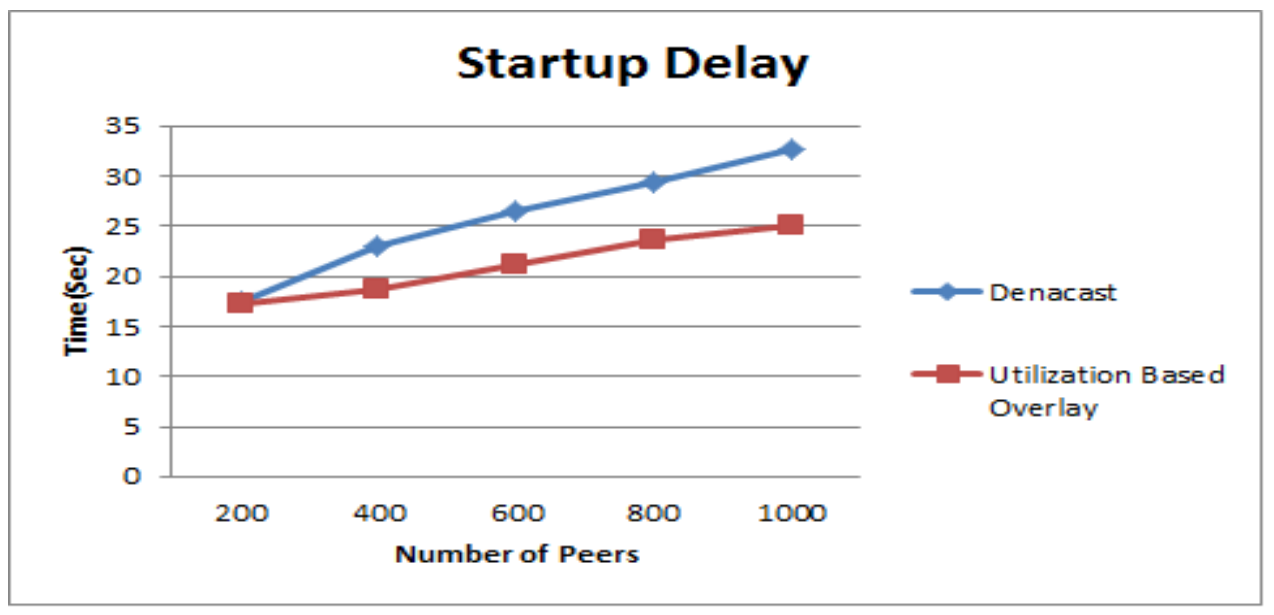

Fig.1: Average Start-up Delay

End to end delay between the peers is also a crucial parameter for P2P networks. If the parent peers are not uploading the content, then the end to end delay at receiver side increases and that degrades the quality of media content which receiver peer wants from its parent. In the utilization based overlay, the parent peers are chosen according to their upload bandwidth and bandwidth utilization; so the peers that are active and have sufficient amount of bandwidth are chosen. This is the reason that why the end to end delay in utilization-based approach is more as compared to that in the DenaCast approach. Fig. 2 shows a comparison of the end to end delay for both the approaches.

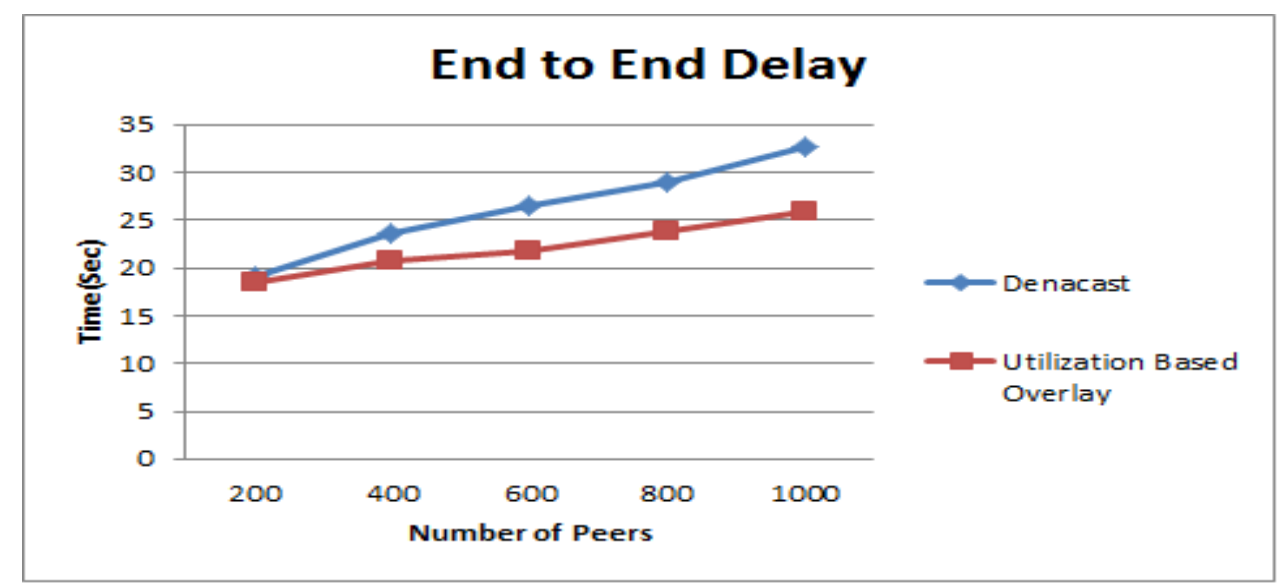

Fig.2: Average End to End Delay

Frame loss ratio of DenaCast approach is more as compared to that for utilization based hybrid overlay approach. As the number of peers increases the frame loss ratio of DenaCast based overlay approach increases while the frame loss ratio in utilization based hybrid overlay approach is less. The reason of frame loss ratio in the P2P network is the number of frames which are lost in the network or are dropped due to late arrival. Frames which arrive at the receiver side after the deadline are useless and just increase the congestion in the network. 
Utilization-based hybrid overlay approach gives priority to peers that have greater utilization, so frames are not lost, and the overall frame loss ratio is also less. A comparison of frame loss ratio to DenaCast and utilization based overlay approaches is shown in Fig. 3.

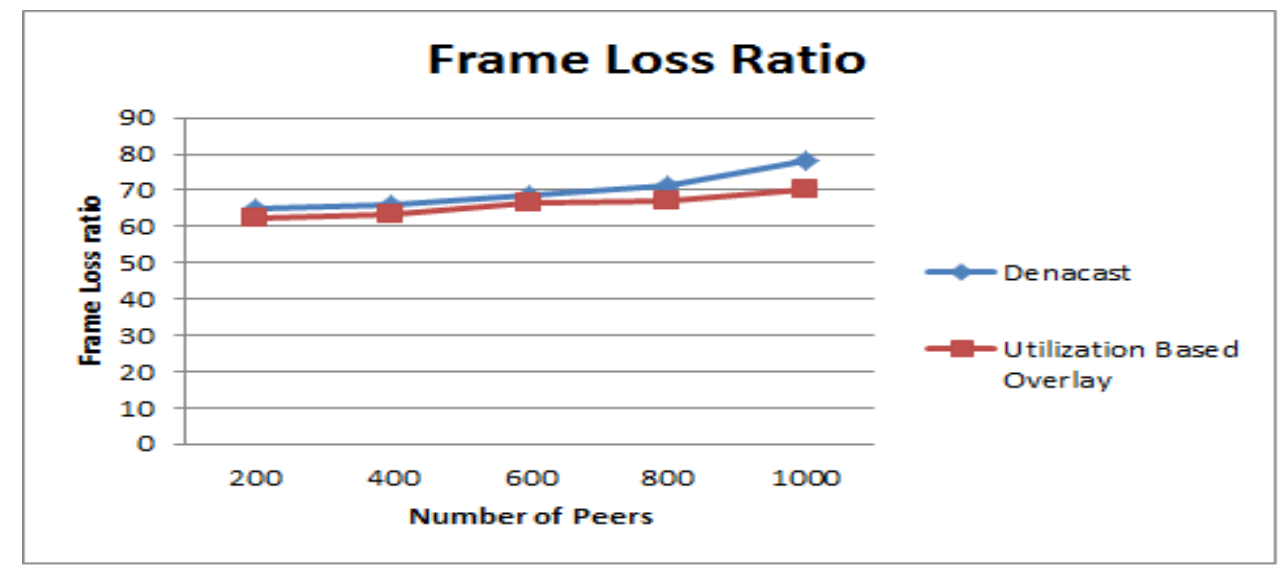

Fig.3: Average Frame Loss Ratio

As the frames are lost or are unreachable to the receiver at the destination due to the expiry of the deadline of the frame, the distortion increases. Due to this reason for frame loss ratio, the distortion of video in utilization based hybrid overlay approach is less compared to that in the DenaCast approach.

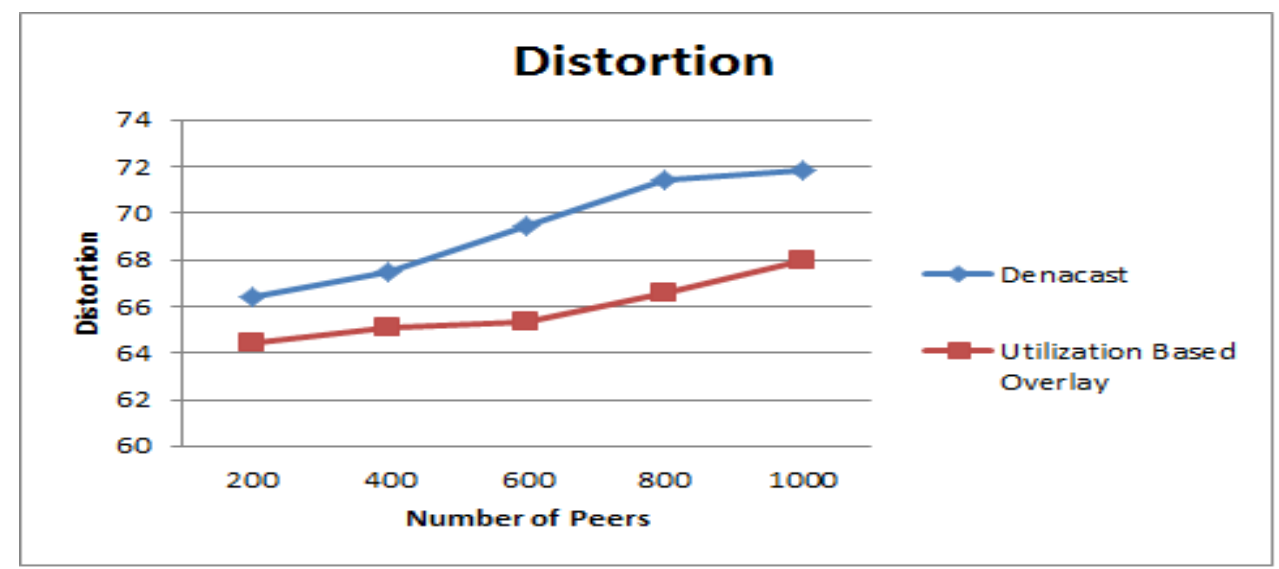

Fig.4: Average Distortion

Playback delay of utilization-based approach is a bit more as compared to that of DenaCast approach. As the number of peers in the network increases it is approximately same for both the approaches and the result is shown in Fig. 5. So overall QoS due to utilization based hybrid overlay increases and the overall performance of the network also increases. 


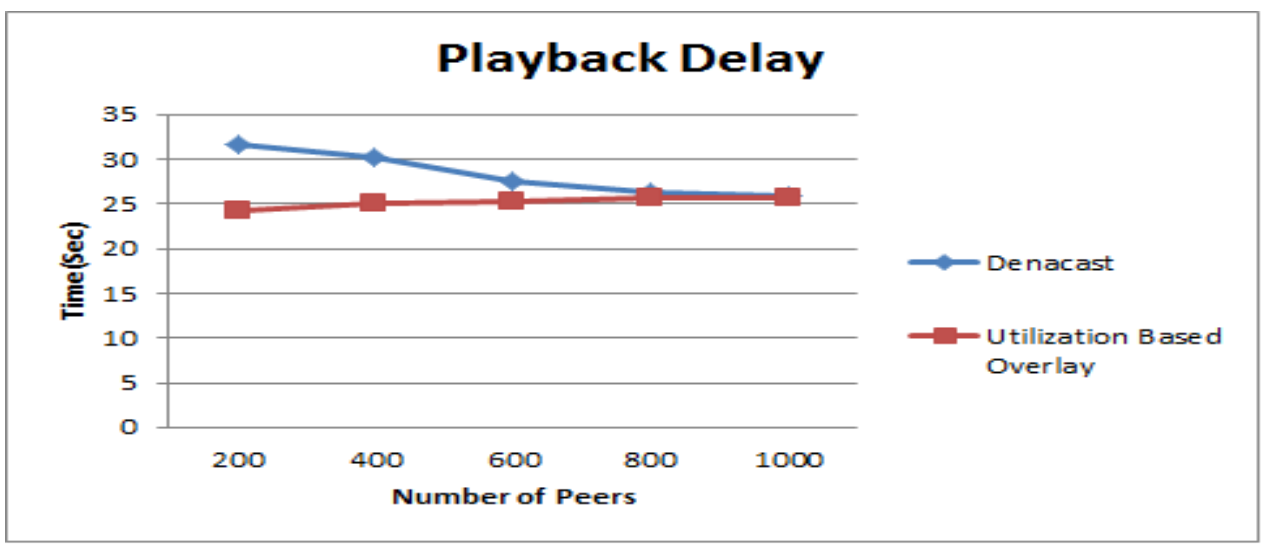

Fig.5: Average Playback Delay

\section{Conclusion and Future Work}

Peer to peer network is gaining popularity for live video streaming transmission due to its scalability, resource utilization, low complexity and cost efficient architecture. However, there are various limitations in the traditional P2P network approaches and; one of them being overlay construction. In this paper, a comparison of different unstructured hybrid overlays for the P2P network is covered, and a new utilization based overlay construction in the $\mathrm{P} 2 \mathrm{P}$ network is proposed. Upload bandwidth and resource utilization of each peer are used for overlay creation. Both the parameters are very useful for overlay creation because, whenever the highly utilized and high upload bandwidth peers are near to the source then it can also provide better media quality to the other peers. Different levels are created for the highly utilized peers in the mesh overlay. Due to these properties of utilization based overlay the QoS of P2P network also improves, and the different QoS parameters also prove the same. The future work aims at providing a detailed comparison of this utilization based hybrid overlay approach with the other different hybrid overlay approaches.

\section{References}

[1] "YouTube." [Online]. Available: https://www.youtube.com/. [Accessed: 11-Jan-2017].

[2] "NetTv." [Online]. Available: http://nettv.com.np/nettv/. [Accessed: 11-Jan-2017].

[3] "IPTV." [Online]. Available: https://www.iptvonline.ca/. [Accessed: 11-Jan-2017].

[4] E. Summary, "Cisco Visual Networking Index: Forecast and Methodology, 2014-2019 White Paper white_paper_c11-481360.pdf," 2015.

[5] S. M. Y. Seyyedi and B. Akbari, "Hybrid CDN-P2P architectures for live video streaming: Comparative study of connected and unconnected meshes," in 2011 International Symposium on Computer Networks and Distributed Systems, CNDS 2011, 2011, pp. 175-180. https://doi.org/10.1109/CNDS.2011.5764567

[6] X. Zhang, J. Liu, B. Li, and T. S. P. Yum, "CoolStreaming/DONet: A data-driven overlay network for efficient live media streaming," Proc. IEEE Infocom, vol. 3, no. C, pp. 13-17, 2005.

[7] L. D'Acunto, T. Vinko, and J. Pouwelse, "Do BitTorrent-like VoD systems scale under flash-crowds?," 2010 IEEE 10th Int. Conf. Peer-to-Peer Comput. P2P 2010 - Proc., no. Section IV, pp. 1-4, 2010. https://doi.org/10.1109/p2p.2010.5569998

[8] S. Xie, B. Li, S. Member, G. Y. Keung, X. Zhang, and S. Member, "Coolstreaming : Design, Theory, and Practice," IEEE Trans. Multimed., vol. 9, no. 8, pp. 1661-1671, 2007. https://doi.org/10.1109/TMM.2007.907469

[9] B. Li et al., "An empirical study of the coolstreaming plus system," IEEE J. Sel. Areas Commun., vol. 25, no. 9, pp. 1627-1639, 2007. https://doi.org/10.1109/JSAC.2007.071203

[10] S. Awiphan, Z. Su, and J. Katto, "ToMo: A two-layer mesh/tree structure for live streaming in P2P overlay 
network," in 2010 7th IEEE Consumer Communications and Networking Conference, CCNC 2010, 2010, pp. 1-5. https://doi.org/10.1109/ccnc.2010.5421709

[11] C. Hammami, I. Jemili, A. Gazdar, A. Belghith, and M. Mosbah, "Hybrid live P2P streaming protocol," Procedia Comput. Sci., vol. 32, pp. 158-165, 2014.

https://doi.org/10.1016/j.procs.2014.05.410

[12] H. B. H. Byun and M. L. M. Lee, "HyPO: A Peer-to-Peer based hybrid overlay structure," in 2009 11th International Conference on Advanced Communication Technology, 2009, vol. 1, pp. 840-844.

[13] N. Magharei and R. Rejaie, "Understanding Mesh-based Peer-to-Peer Streaming," in Proceedings of the 2006 international workshop on Network and operating systems support for digital audio and video., 2006, p. 10. https://doi.org/10.1145/1378191.1378204

[14] N. Magharei and R. Rejaie, "PRIME : Peer-to-Peer Receiver-drIven MEsh-based Streaming," pp. 1415-1423, 2007.

[15] Q. Huang, H. Jin, and X. Liao, "P2P live streaming with tree-mesh based hybrid overlay," Proc. Int. Conf. Parallel Process. Work., no. 60433040, 2007.

https://doi.org/10.1109/icppw.2007.65

[16] P. K. Govil M.C, Ahmed Mushtaq, "A New Hybrid Approach for Overlay Construction in P2P Live Streaming," in ICACCI, 2015, pp. 431-437.

[17] P. K. Govil M.C, Ahmed Mushtaq, "Comparative Analysis of New Hybrid Approach for Overlay Construction in P2P Live Streaming," in ERCICA, 2016.

[18] I. Baumgart, I. Baumgart, B. Heep, B. Heep, S. Krause, and S. Krause, "OverSim: A Flexible Overlay Network Simulation Framework," 2007 IEEE Glob. Internet Symp., pp. 79-84, 2007.

https://doi.org/10.1109/GI.2007.4301435 